\title{
Initial boundary value problem for a class of higher-order $n$-dimensional nonlinear pseudo-parabolic equations
}

\author{
Liming Xiao ${ }^{1 *}$ (D) and Mingkun Li
}

\section{"Correspondence:}

13711738661@163.com

'School of Mathematics and

Systems Science, Guangdong

Polytechnic Normal University,

Guangzhou 510665, P.R. China

\section{Springer}

\begin{abstract}
In this paper, we study the initial boundary value problem for a class of higher-order n-dimensional nonlinear pseudo-parabolic equations which do not have positive energy and come from the soil mechanics, the heat conduction, and the nonlinear optics. By the mountain pass theorem we first prove the existence of nonzero weak solution to the static problem, which is the important basis of evolution problem, then based on the method of potential well we prove the existence of global weak solution to the evolution problem.
\end{abstract}

MSC: 35G31; 35D30; 35D35; 35B45

Keywords: Nonlinear pseudo-parabolic equation; Higher-order; The method of potential well; Static problem; Existence of global weak solution

\section{Introduction}

In this paper, we consider the following initial boundary value problem for $n$-dimensional nonlinear pseudo-parabolic equations of arbitrary higher order:

$$
\begin{aligned}
& u_{t}(x, t)+(-1)^{M} \Delta^{M} u_{t}(x, t)+(-1)^{M} \Delta^{M} u(x, t)=f(u(x, t)), \quad x \in \Omega, t \in[0, T), \\
& u(x, 0)=u_{0}(x), \quad x \in \Omega, \\
& \left.D^{\gamma} u(x, t)\right|_{\partial \Omega \times[0, T)}=0, \quad 0 \leq|\gamma| \leq M-1,
\end{aligned}
$$

where $\Omega \subset \mathbb{R}^{n}$ is a bounded domain with sufficiently smooth boundary $\partial \Omega, M$ is a positive integer, $\gamma=\left(\gamma_{1}, \gamma_{2}, \ldots, \gamma_{n}\right)$ is multi-index, $\gamma_{i}(i=1,2, \ldots, n)$ are nonnegative integers, $|\gamma|=$ $\gamma_{1}+\gamma_{2}+\cdots+\gamma_{n}, D^{\gamma}=\frac{\partial|\gamma|}{\partial x_{1}^{\gamma 1} \partial x_{2}^{\gamma 2} \cdots \partial x_{n}^{\gamma / n}}$ means multi-index derivative operator, $\Delta=\sum_{i=1}^{n} \frac{\partial^{2}}{\partial x_{i}^{2}}$ is an $n$-dimensional Laplace operator, $u_{0}(x) \in H^{2 M}(\Omega) \cap H_{0}^{M}(\Omega), H^{2 M}(\Omega)$, and $H_{0}^{M}(\Omega)$ are Sobolev spaces. Moreover, $f(u)=a|u|^{p-1} u$ and $f(\cdot)$ satisfies condition $(\mathrm{H}): f(u) u \geq 0$, where $a>0, p>1$ such that

$$
\text { if } \quad 2 M \geq n, \quad 1<p<+\infty ; \quad \text { if } \quad 2 M<n, \quad 1<p \leq \frac{n}{n-2 M} .
$$

(c) The Author(s) 2021. This article is licensed under a Creative Commons Attribution 4.0 International License, which permits use, sharing, adaptation, distribution and reproduction in any medium or format, as long as you give appropriate credit to the original author(s) and the source, provide a link to the Creative Commons licence, and indicate if changes were made. The images or other third party material in this article are included in the article's Creative Commons licence, unless indicated otherwise in a credit line to the material. If material is not included in the article's Creative Commons licence and your intended use is not permitted by statutory regulation or exceeds the permitted use, you will need to obtain permission directly from the copyright holder. To view a copy of this licence, visit http://creativecommons.org/licenses/by/4.0/. 
The inequality $f(u) u \geq 0$ means that the direction of external force is the same as the direction of displacement.

Problem (1.1)-(1.3) describes some important physical issues (see [2]) and has attracted the attention of many scholars. For example, Benedetto, Pierre, Gopala Rao, Slowalter, and Ting $[1,5,20,22]$ considered the initial boundary value problem for the equation

$$
u_{t}-k \Delta u_{t}-\Delta u=0
$$

got the maximum principle and established the existence and uniqueness of solution. Cao, Yin, and Wang in [3] studied the Cauchy problem of semilinear pseudo-parabolic equations. With the initial data being appropriately smooth, they got the existence and uniqueness of mild solution. Moreover, they proved that there exist the critical global existence exponent and the critical Fujita exponent for pseudo-parabolic equations.

For the equation

$$
u_{t}-\Delta u_{t}-\Delta u=u^{p}
$$

$\mathrm{Xu}$ and $\mathrm{Su}$ in [26] considered the initial boundary value problem and proved the global existence, nonexistence, and asymptotic behavior of solutions when $J\left(u_{0}\right) \leq d$. Moreover, they proved finite time blow-up when $J\left(u_{0}\right)>d$ by the comparison principle. For the background of the problem in [26], one can refer to [7, 8, 14, 21].

Up to now, there has been no paper to study the nonlinear evolution equations of arbitrary higher order by the method of potential well, and the study for nonlinear evolution equations of arbitrary higher order by the method of potential well is more difficult. If $M=1$, there have been some results about the initial boundary value problem (1.1)-(1.3) (see [26]). If $M \geq 2$, there have not been any results about the initial boundary value problem (1.1)-(1.3). In this paper, for $M \in N^{+}=\{1,2,3, \ldots\}$, the initial boundary value problem (1.1)-(1.3) is studied by the method of potential well. In the growth condition of nonlinear term $f(u)$, the growth exponent $p$ depends not only on space dimension $n$, but also on $M$ (see condition $(\mathrm{H})$ ). The result of this paper is applicable for a big class of higher-order $n$-dimensional nonlinear pseudo-parabolic equations. Moreover, the study for the higherorder nonlinear evolution equation will be related to the properties about the higher-order eigenfunctions and the higher-order eigenvalues of Laplace operator.

The method of potential well was proposed by Sattinger in [19] and was used to prove the existence of global weak solutions to nonlinear hyperbolic equations which do not have positive energy. Payne and Sattinger in [18] have shown the theory of potential well in detail and made the further study of potential well. Under some conditions they have proved that the solutions to the equation $u_{t t}-\Delta u=f(u)(x \in \Omega, t>0)$ will blow up in finite time.

Recently, the potential well method has been well applied in some important physical problems. Liu and Zhao in [13] generalized the family of potential wells to the initial boundary value problems of semilinear hyperbolic equations and parabolic equations. They not only gave a threshold result of global existence and nonexistence of solutions, but also obtained the vacuum isolating of solutions. On the basis of Liu and Zhao's work, $\mathrm{Xu}$ in [24] continued to study the initial boundary value problem with critical initial data $E(0)=d\left(\right.$ or $\left.J\left(u_{0}\right)=d\right), I\left(u_{0}\right)<0$ and proved that there exist non-global solutions under 
the classical conditions on $f$. By the method of potential well, $\mathrm{Liu}, \mathrm{Xu}$, and $\mathrm{Yu}$ in [12] studied the Cauchy problem of semilinear heat equations and obtained a threshold result for the global existence and nonexistence of solutions. Moreover, they got the asymptotic behavior of the solution when $J\left(u_{0}\right) \leq d$. Xu in [23] discussed the initial boundary value problem of semilinear parabolic equations with semilinear term $f(u)$. By introducing a family of potential wells, not only the solutions decay to zero with $f(u)$ satisfying some conditions when $J\left(u_{0}\right) \leq d$ and $I\left(u_{0}\right)>0$ was proved, but also finite time blows up when $J\left(u_{0}\right) \leq d$ and $I\left(u_{0}\right)<0$ was obtained. In addition, based on the potential well method, for the case of high initial energy, finite time blow-up was proved (see [9, 25, 27]); for dealing with problems with variational methods, one can also refer to $[15,16]$. In fact, there are many well-known results, which affect both global existence and finite time blow-up of solutions to the nonlinear damping wave equations, if we focus on the initial data (see $[10,17,28,29])$.

This paper is organized as follows.

(i) In Sect. 2: we study the static problem which is closely related to the evolution problem and is the important basis of the evolution problem.

(ii) In Sect. 3: some useful lemmas and their proofs are given.

(iii) In Sect. 4: by using the method of potential well, we prove the existence of global weak solutions to problem (1.1)-(1.3) with subcritical energy, i.e., $E(0)<d$, which is shown in Theorem 4.1.

Throughout the paper, we denote $\|\cdot\|_{L^{p}(\Omega)}=\|\cdot\|_{p},\|\cdot\|_{L^{2}(\Omega)}=\|\cdot\|,\|\cdot\|_{W^{k, p}(\Omega)}=\|\cdot\|_{k, p}$, $(u(x), v(x))=\int_{\Omega} u(x) v(x) d x$, and $[u(x, t), v(x, t)]=\int_{0}^{t}(u(x, \tau), v(x, \tau)) d \tau$.

Let us begin with defining the following total energy functional:

$$
E(t)=\frac{1}{2}\left\|\nabla^{M} u(x, t)\right\|^{2}-\int_{\Omega} F(u(x, t)) d x,
$$

where $\nabla=\left(\frac{\partial}{\partial x_{1}}, \frac{\partial}{\partial x_{2}}, \ldots, \frac{\partial}{\partial x_{n}}\right)$ is an $n$-dimensional gradient operator and $F(u)=\int_{0}^{u} f(s) d s$.

Then the potential energy functional

$$
J(u)=\frac{1}{2}\left\|\nabla^{M} u(x, t)\right\|^{2}-\frac{a}{p+1}\|u(x, t)\|_{p+1}^{p+1}
$$

and the Nehari functional

$$
I(u)=\left\|\nabla^{M} u(x, t)\right\|^{2}-a\|u(x, t)\|_{p+1}^{p+1} .
$$

By $I(u)$ we define the Nehari manifold

$$
\mathcal{N}=\left\{u(x, t) \in H_{0}^{M}(\Omega) \mid I(u)=0,\left\|\nabla^{M} u\right\| \neq 0\right\},
$$

the potential well

$$
W=\left\{u(x, t) \in H_{0}^{M}(\Omega) \mid I(u)>0, J(u)<d\right\} \cup\{0\},
$$

and the set outside the potential well

$$
V=\left\{u(x, t) \in H_{0}^{M}(\Omega) \mid I(u)<0, J(u)<d\right\},
$$


where $d$ is the depth of the potential well which is defined as follows:

$$
\begin{aligned}
d & =\inf _{\substack{u(x) \in H_{0}^{M}(\Omega), u \neq 0}}\left(\sup _{\lambda \geq 0} J(\lambda u)\right) \\
& =\inf _{u \in \mathcal{N}} J(u) .
\end{aligned}
$$

\section{Static problem}

In this section, the existence of nonzero weak solution to the following boundary value problem

$$
\left\{\begin{array}{l}
(-1)^{M} \Delta^{M} u=f(u), \quad x \in \Omega \subset \mathbb{R}^{n}, \\
\left.D^{\gamma} u(x)\right|_{\partial \Omega}=0, \quad 0 \leq|\gamma| \leq M-1
\end{array}\right.
$$

is considered. This is the important basis of evolution problem, where $\Omega, M, \Delta, \gamma$, and $D^{\gamma}$ are the same as in Sect. 1.

Assume that the function $f(\cdot)$ satisfies: $f \in C^{1}, f(u) u \geq 0$ (the condition can conclude that $\left.F(u) \triangleq \int_{0}^{u} f(s) d s \geq 0\right)$ and

$$
b|u|^{p} \leq|f(u)| \leq a|u|^{p},\left|f^{\prime}(u)\right| \leq a_{1}|u|^{p-1},
$$

where $a>b>0, a_{1}>0, a, b$, and $a_{1}$ are positive real constants. In addition, the positive real number $p$ satisfies that when $2 M<n, 1<p<\frac{n+2 M}{n-2 M}$; when $2 M \geq n, 1<p<+\infty$.

Assume that there exists $0<\gamma<\frac{1}{2}$ such that

$$
0 \leq F(u) \leq \gamma f(u) u
$$

and

$$
b^{\prime}|u|^{p+1} \leq|F(u)| \leq a^{\prime}|u|^{p+1},
$$

where $a^{\prime}$ and $b^{\prime}$ are positive real constants $\left(a^{\prime}>b^{\prime}\right)$.

Take $f(u)=\frac{1}{4} u^{2 m+1}$, where $m$ is a positive integer. Then the above conditions can be satisfied.

Definition 2.1 The function $u(x) \in H_{0}^{M}(\Omega)$ is known as the weak solution to problem (2.1). If, for each $v(x) \in H_{0}^{M}(\Omega)$, we have

$$
\int_{\Omega} \nabla^{M} u(x) \nabla^{M} v(x) d x-\int_{\Omega} f(u(x)) v(x) d x=0 .
$$

Obviously, $u=0$ is the trivial weak solution to problem (2.1). In what follows we will prove that there exists a nontrivial weak solution to problem (2.1).

Make the functional

$$
I(u)=\frac{1}{2} \int_{\Omega}\left|\nabla^{M} u(x)\right|^{2} d x-\int_{\Omega} F(u(x)) d x, \quad u(x) \in H_{0}^{M}(\Omega) .
$$


First, we will prove that if $I\left(u_{0}(x)\right)=\min _{u \in H_{0}^{M}(\Omega)} I(u)$, the function $u_{0}(x)$ must be the weak solution to problem (2.1).

Lemma 2.1 If $I\left(u_{0}(x)\right)=\min _{u \in H_{0}^{M}(\Omega)} I(u)$, then the function $u_{0}(x)$ must be the weak solution to problem (2.1).

Proof Assume that the functional $I(u)$ takes its minimum at $u_{0}(x) \in H_{0}^{M}(\Omega)$, it concludes that $\forall h(x) \in H_{0}^{M}(\Omega)$, the function $\varphi(t)=I\left(u_{0}(x)+t h(x)\right)$ takes its minimum at $t=0$, that is, $\varphi^{\prime}(0)=0$.

Make the following computations:

$$
\begin{aligned}
\varphi(t)= & I\left(u_{0}(x)+\operatorname{th}(x)\right) \\
= & \frac{1}{2} \int_{\Omega}\left|\nabla^{M}\left(u_{0}(x)+t h(x)\right)\right|^{2} d x-\int_{\Omega} F\left(u_{0}(x)+t h(x)\right) d x \\
= & \frac{1}{2} \int_{\Omega} \nabla^{M} u_{0}(x) \nabla^{M} u_{0}(x) d x+t \int_{\Omega} \nabla^{M} u_{0}(x) \nabla^{M} h(x) d x \\
& +\frac{t^{2}}{2} \int_{\Omega} \nabla^{M} h(x) \nabla^{M} h(x) d x-\int_{\Omega} F\left(u_{0}(x)+t h(x)\right) d x,
\end{aligned}
$$

therefore,

$$
\varphi^{\prime}(t)=\int_{\Omega} \nabla^{M} u_{0}(x) \nabla^{M} h(x) d x+t \int_{\Omega} \nabla^{M} h(x) \nabla^{M} h(x) d x-\int_{\Omega} f\left(u_{0}(x)+t h(x)\right) h(x) d x .
$$

By $\varphi^{\prime}(0)=0$, we conclude that

$$
\int_{\Omega} \nabla^{M} u_{0}(x) \nabla^{M} h(x) d x-\int_{\Omega} f\left(u_{0}(x)\right) h(x) d x=0, \quad \forall h(x) \in H_{0}^{M}(\Omega) .
$$

Hence, for each $h(x) \in H_{0}^{M}(\Omega)$, we get

$$
\begin{aligned}
& \left(I^{\prime}\left(u_{0}(x)\right), h(x)\right)_{M} \\
& \quad \triangleq \int_{\Omega} \nabla^{M} u_{0}(x) \nabla^{M} h(x) d x-\int_{\Omega} f\left(u_{0}(x)\right) h(x) d x \\
& \quad=0 .
\end{aligned}
$$

The function $u_{0}(x) \in H_{0}^{M}(\Omega)$ is the weak solution to problem (2.1).

The proof of Lemma 2.1 is completed.

From the above discussion we know that if we hope to find the nontrivial weak solution to problem (2.1), we need to find the nonzero minimum point of the functional $I(u)$ in $H_{0}^{M}(\Omega)$. We will prove the conclusion by the mountain pass theorem.

Notice that $\left\|\nabla^{M} u(x)\right\|_{L^{2}(\Omega)}$ is an equivalent norm in $H_{0}^{M}(\Omega)$, we can define the inner product in $H_{0}^{M}(\Omega)$ : for $u(x), v(x) \in H_{0}^{M}(\Omega)$,

$$
(u(x), v(x))_{M}=\int_{\Omega} \nabla^{M} u(x) \nabla^{M} v(x) d x .
$$


By the above inner product we can induce the following norm:

$$
\left[(u(x), u(x))_{M}\right]^{\frac{1}{2}}=\left[\int_{\Omega}\left|\nabla^{M} u(x)\right|^{2} d x\right]^{\frac{1}{2}} \triangleq\|u(x)\|_{M},
$$

which is an equivalent norm in $H_{0}^{M}(\Omega)$.

The following Theorem 2.1 shows that the functional $I(u)$ has a nonzero minimum point in $H_{0}^{M}(\Omega)$.

Theorem 2.1 If the function $f \in C^{1}$ and the inequality $f(u) u \geq 0$ hold; moreover, if the three inequalities (2.2), (2.3), and (2.4) hold. Then the functional $I(u)$ has a nonzero minimum point in $H_{0}^{M}(\Omega)$.

Proof We first consider the case when $2 M<n$.

Step 1: We will verify that $I(u)$ is a $C^{1}$ functional in $H_{0}^{M}(\Omega)$. For each $u(x) \in H_{0}^{M}(\Omega)$, we have

$$
\begin{aligned}
I(u) & =\frac{1}{2} \int_{\Omega}\left|\nabla^{M} u(x)\right|^{2} d x-\int_{\Omega} F(u) d x \\
& =I_{1}(u)-I_{2}(u),
\end{aligned}
$$

where $I_{1}(u)=\frac{1}{2} \int_{\Omega}\left|\nabla^{M} u(x)\right|^{2} d x$ and $I_{2}(u)=\int_{\Omega} F(u) d x$.

For $u(x), w(x) \in H_{0}^{M}(\Omega)$, by some computations,

$$
\begin{aligned}
I_{1}(w(x)) & =\frac{1}{2} \int_{\Omega}\left|\nabla^{M} w(x)\right|^{2} d x \\
& =\frac{1}{2}\|w(x)\|_{M}^{2} \\
& =\frac{1}{2}\|u(x)+w(x)-u(x)\|_{M}^{2} \\
& =\frac{1}{2}\|u(x)\|_{M}^{2}+(u(x), w(x)-u(x))_{M}+\frac{1}{2}\|w(x)-u(x)\|_{M}^{2} .
\end{aligned}
$$

Therefore, the functional $I_{1}$ is differential at $u(x)$ and $I_{1}^{\prime}(u)=u$. It concludes that $I_{1}(u)$ is a $C^{1}$ functional in $H_{0}^{M}(\Omega)$.

In what follows we will prove that the functional $I_{2}(u)$ is also a $C^{1}$ functional in $H_{0}^{M}(\Omega)$. For each $v^{*} \in H^{-M}(\Omega)=\left(H_{0}^{M}(\Omega)\right)^{*}$, by the Lax-Milgram theorem the problem

$$
\left\{\begin{array}{l}
(-1)^{M} \Delta^{M} v=v^{*}, \quad \text { in } \Omega, \\
\left.D^{\gamma} v\right|_{\partial \Omega}=0, \quad 0 \leq|\gamma| \leq M-1
\end{array}\right.
$$

has only one weak solution $v(x) \in H_{0}^{M}(\Omega)$.

Denote $v(x)=K v^{*}, K: H^{-M}(\Omega) \rightarrow H_{0}^{M}(\Omega)$ is an isometry mapping, that is, for each $v^{*} \in$

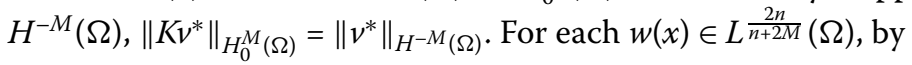

$$
\left\langle w^{*}, v\right\rangle=\int_{\Omega} w v d x, \quad v(x) \in H_{0}^{M}(\Omega)
$$

we can define a bounded linear functional $w^{*} \in H^{-M}(\Omega)=\left(H_{0}^{M}(\Omega)\right)^{*}$. 
By $2 M<n$ and $1<p<\frac{n+2 M}{n-2 M}$, it concludes that $\frac{2 n p}{n+2 M}<\frac{2 n}{n-2 M} \triangleq 2_{M}^{*}$. From $|f(u)|<a|u|^{p}$ we can get that

$$
|f(u)|^{\frac{2 n}{n+2 M}}<a^{\frac{2 n}{n+2 M}}|u|^{\frac{2 n p}{n+2 M}}
$$

Hence

$$
\int_{\Omega}|f(u)|^{\frac{2 n}{n+2 M}} d x<a^{\frac{2 n}{n+2 M}} \int_{\Omega}|u|^{\frac{2 n p}{n+2 M}} d x .
$$

By $u(x) \in H_{0}^{M}(\Omega), \frac{2 n p}{n+2 M}<2_{M}^{*}$ and the Sobolev imbedding theorem, we can conclude that $\int_{\Omega}|u|^{\frac{2 n p}{n+2 M}} d x<+\infty$, therefore $\int_{\Omega}|f(u)|^{\frac{2 n}{n+2 M}} d x<+\infty$, that is, $f(u) \in L^{\frac{2 n}{n+2 M}}(\Omega)$.

From the above discussion it shows that by $f(u)$ we can define a bounded linear functional $f(u) \in H^{-M}(\Omega)=\left(H_{0}^{M}(\Omega)\right)^{*}$. Since $K$ is an isometry mapping from $H^{-M}(\Omega)$ to $H_{0}^{M}(\Omega)$, it concludes that $K(f(u)) \in H_{0}^{M}(\Omega)$. Because $K(f(u))$ is a weak solution to the following problem:

$$
\left\{\begin{array}{l}
(-1)^{M} \Delta^{M} v=f(u), \\
\left.D^{\gamma} v\right|_{\partial \Omega}=0, \quad 0 \leq|\gamma| \leq M-1,
\end{array}\right.
$$

by the Lax-Milgram theorem, for each $h(x) \in H_{0}^{M}(\Omega)$, we have

$$
\int_{\Omega} \nabla^{M}(K f(u)) \nabla^{M} h(x) d x=\int_{\Omega} f(u) h(x) d x .
$$

In what follows we prove that, for each $u(x) \in H_{0}^{M}(\Omega)$, we can get

$$
I_{2}^{\prime}(u)=K(f(u))
$$

For $a, b \in \mathbb{R}$, by some computations, we derive

$$
F(a+b)=F(a)+f(a) b+b^{2} \int_{0}^{1}(1-s) f^{\prime}(a+b s) d s .
$$

By equality (2.5), for each $w(x) \in H_{0}^{M}(\Omega)$,

$$
\begin{aligned}
I_{2}(w) & =\int_{\Omega} F(w) d x \\
& =\int_{\Omega} F(u+w-u) d x \\
& =\int_{\Omega}[F(u)+f(u)(w-u)] d x+R \\
& =I_{2}(u)+\int_{\Omega} f(u)(w-u) d x+R \\
& =I_{2}(u)+\int_{\Omega} \nabla^{M}\left(K(f(u)) \nabla^{M}(w-u) d x+R\right. \\
& =I_{2}(u)+(K(f(u)), w-u)_{M}+R,
\end{aligned}
$$

where $R=\int_{\Omega} \int_{0}^{1}(1-s) f^{\prime}(u+s(w-u)) d s(w-u)^{2} d x$. 
In what follows we make some estimates about $R$.

$$
\begin{aligned}
|R| & \leq \int_{\Omega} \int_{0}^{1}\left|(1-s) f^{\prime}(u+s(w-u)) d s(w-u)^{2}\right| d x \\
& \leq \int_{\Omega} \int_{0}^{1}\left|f^{\prime}(u+s(w-u))\right| d s(w-u)^{2} d x \\
& \leq \int_{\Omega} \int_{0}^{1} a_{1}|u+s(w-u)|^{p-1} d s(w-u)^{2} d x \\
& \leq \int_{\Omega} \int_{0}^{1} C\left(|u|^{p-1}+|w-u|^{p-1}\right) d s(w-u)^{2} d x \\
& =C \int_{\Omega}|u|^{p-1}|w-u|^{2} d x+C \int_{\Omega}|w-u|^{p+1} d x
\end{aligned}
$$

where $p+1<\frac{n+2 M}{n-2 M}+1=2_{M}^{*}$. To save notations, in the following discussion $C$ represents different positive constants in different places.

Since $w(x), u(x) \in H_{0}^{M}(\Omega)$, by the Sobolev imbedding theorem, there exists a positive constant $C$ such that

$$
\|w-u\|_{L^{p+1}(\Omega)}^{p+1} \leq C\|w-u\|_{H_{0}^{M}(\Omega)}^{p+1} \leq C\|w-u\|_{M}^{p+1}
$$

where $\|w-u\|_{M}=\left[\int_{\Omega}\left|\nabla^{M}(w-u)\right|^{2} d x\right]^{\frac{1}{2}}$.

By Hölder's inequality, we have

$$
\begin{aligned}
& \int_{\Omega}|u|^{p-1}|w-u|^{2} d x \\
& \quad \leq\left\{\int_{\Omega}|u|^{p+1} d x\right\}^{\frac{p-1}{p+1}}\left\{\int_{\Omega}|w-u|^{p+1} d x\right\}^{\frac{2}{p+1}} \\
& \quad=\|u\|_{L^{p+1}(\Omega)}^{p-1}\|w-u\|_{L^{p+1}(\Omega)}^{2} .
\end{aligned}
$$

Notice that $p+1<\frac{n+2 M}{n-2 M}+1=2_{M}^{*}$, by $u(x), w(x) \in H_{0}^{M}(\Omega)$, and the Sobolev imbedding theorem, we can get that

$$
\|w-u\|_{L^{p+1}(\Omega)}^{2} \leq C\|w-u\|_{H_{0}^{M}(\Omega)}^{2} \leq C\|w-u\|_{M}^{2} .
$$

Since $u(x) \in H_{0}^{M}(\Omega)$, it concludes that

$$
\|u\|_{L^{p+1}(\Omega)}^{p-1} \leq C
$$

Therefore,

$$
|R| \leq C\|w-u\|_{M}^{p+1}+C\|w-u\|_{M}^{2},
$$

that is, $R=o\left(\|w-u\|_{M}\right)$.

By the above conclusions we can get that

$$
I_{2}(w)=I_{2}(u)+(K(f(u)), w-u)_{M}+R,
$$


where $R=o\left(\|w-u\|_{M}\right)$. By the definition of Fréchet derivative,

$$
I_{2}^{\prime}(u)=K(f(u))
$$

In what follows we will prove that $I_{2}^{\prime}: H_{0}^{M}(\Omega) \rightarrow H_{0}^{M}(\Omega)$ is Lipschitz continuous on a bounded subset of $H_{0}^{M}(\Omega)$.

Assume that $u(x), \bar{u}(x) \in H_{0}^{M}(\Omega)$ and $u(x), \bar{u}(x)$ satisfy $\|u(x)\|_{H_{0}^{M}(\Omega)} \leq L$ and $\|\bar{u}(x)\|_{H_{0}^{M}(\Omega)} \leq L$, where $L$ is a positive constant. Then we have

$$
\begin{aligned}
& \left\|I_{2}^{\prime}(u)-I_{2}^{\prime}(\bar{u})\right\|_{M} \\
& \quad \leq C\left\|I_{2}^{\prime}(u)-I_{2}^{\prime}(\bar{u})\right\|_{H_{0}^{M}(\Omega)} \\
& \quad=C\|K(f(u))-K(f(\bar{u}))\|_{H_{0}^{M}(\Omega)}
\end{aligned}
$$

( $K$ is an isometry mapping from $H^{-M}(\Omega)$ to $H_{0}^{M}(\Omega)$ )

$$
\begin{aligned}
& =C\|f(u)-f(\bar{u})\|_{H^{-M}(\Omega)} \\
& \leq C\|f(u)-f(\bar{u})\|_{L^{\frac{2 n}{n+2 M}(\Omega)}} \\
& =C\left\{\int_{\Omega}|f(u)-f(\bar{u})|^{\frac{2 n}{n+2 M}} d x\right\}^{\frac{n+2 M}{2 n}} \\
& \leq C\left\{\int_{\Omega}\left(|\bar{u}|^{p-1}+|u|^{p-1}\right)^{\frac{n}{2 M}} d x\right\}^{\frac{2 M}{n}}\left\{\int_{\Omega}|u-\bar{u}|^{\frac{2 n}{n-2 M}} d x\right\}^{\frac{n-2 M}{2 n}} \\
& =C\left\{\int_{\Omega}\left(|\bar{u}|^{p-1}+|u|^{p-1}\right)^{\frac{n}{2 M}} d x\right\}^{\frac{2 M}{n}}\|u-\bar{u}\|_{L^{2} M(\Omega)}
\end{aligned}
$$

Since $\frac{n(p-1)}{2 M}<\left(\frac{n+2 M}{n-2 M}-1\right) \frac{n}{2 M}=2_{M}^{*}$, by $\|u(x)\|_{H_{0}^{M}(\Omega)} \leq L,\|\bar{u}(x)\|_{H_{0}^{M}(\Omega)} \leq L$, and the Sobolev imbedding theorem, we can get that

$$
\left\{\int_{\Omega}\left(|\bar{u}|^{p-1}+|u|^{p-1}\right)^{\frac{n}{2 M}} d x\right\}^{\frac{2 M}{n}} \leq C
$$

Therefore,

$$
\begin{aligned}
& \|f(u)-f(\bar{u})\|_{L^{\frac{2 n}{n+2 M}(\Omega)}} \\
& \leq C\|u-\bar{u}\|_{L^{2_{M}^{*}(\Omega)}} \\
& \leq C\|u-\bar{u}\|_{H_{0}^{M}(\Omega)} \\
& \leq C\|u-\bar{u}\|_{M} .
\end{aligned}
$$

Summarizing the above discussion, there exists a positive constant $C$ such that

$$
\left\|I_{2}^{\prime}(u)-I_{2}^{\prime}(\bar{u})\right\|_{M} \leq C\|u-\bar{u}\|_{M} .
$$


Therefore, mapping $I_{2}^{\prime}: H_{0}^{M}(\Omega) \rightarrow H_{0}^{M}(\Omega)$ is Lipschitz continuous on a bounded subset of $H_{0}^{M}(\Omega)$, it means that $I_{2}$ is a $C^{1}$ functional. Because $I_{1}$ is also a $C^{1}$ functional, $I(u)=$ $I_{1}(u)-I_{2}(u)$ is a $C^{1}$ functional.

The proof of Step 1 is completed.

Step 2: In what follows we verify that functional $I(u)$ satisfies the Palais-Smale condition. Assume that $\left\{u_{k}(x)\right\}_{k=1}^{+\infty} \subset H_{0}^{M}(\Omega)$ satisfies: $\left|I\left(u_{k}\right)\right| \leq C(k=1,2,3, \ldots)$ and when $k \rightarrow+\infty$, $I^{\prime}\left(u_{k}\right) \rightarrow 0$ in $H_{0}^{M}(\Omega)$. Under the above conditions we will prove that there exists a subsequence $\left\{u_{k_{j}}(x)\right\}_{j=1}^{+\infty}$ of $\left\{u_{k}(x)\right\}_{k=1}^{+\infty}$ such that $\left\{u_{k_{j}}(x)\right\}_{j=1}^{+\infty}$ is strongly convergent in $H_{0}^{M}(\Omega)$.

By the known conditions, we get

$$
\begin{aligned}
C & \geq\left|I\left(u_{k}\right)\right| \\
& \geq I\left(u_{k}\right) \\
& =\frac{1}{2} \int_{\Omega}\left|\nabla^{M} u_{k}(x)\right|^{2} d x-\int_{\Omega} F\left(u_{k}\right) d x \\
& =\frac{1}{2}\left\|u_{k}(x)\right\|_{M}^{2}-\int_{\Omega} F\left(u_{k}\right) d x \\
& \geq \frac{1}{2}\left\|u_{k}(x)\right\|_{M}^{2}-\gamma \int_{\Omega} u_{k} f\left(u_{k}\right) d x \quad\left(0<\gamma<\frac{1}{2}\right) \\
& =\left(\frac{1}{2}-\gamma\right)\left\|u_{k}(x)\right\|_{M}^{2}+\gamma \int_{\Omega}\left[\left(\nabla^{M} u_{k}\right)\left(\nabla^{M} u_{k}\right)-u_{k} f\left(u_{k}\right)\right] d x \\
& =\left(\frac{1}{2}-\gamma\right)\left\|u_{k}(x)\right\|_{M}^{2}+\gamma\left(I^{\prime}\left(u_{k}\right), u_{k}\right)_{M} \\
& \geq\left(\frac{1}{2}-\gamma\right)\left\|u_{k}(x)\right\|_{M}^{2}-\gamma\left\|I^{\prime}\left(u_{k}\right)\right\|_{M}\left\|u_{k}\right\|_{M} .
\end{aligned}
$$

When $k \rightarrow+\infty,\left\|I^{\prime}\left(u_{k}\right)\right\|_{H_{0}^{M}(\Omega)} \rightarrow 0$, it concludes that $\left\|I^{\prime}\left(u_{k}\right)\right\|_{M} \rightarrow 0$. By $0<\gamma<\frac{1}{2}$, the $\left\|I^{\prime}\left(u_{k}\right)\right\|_{M}$ is bounded with respect to $k$ and the inequality

$$
C \geq\left(\frac{1}{2}-\gamma\right)\left\|u_{k}(x)\right\|_{M}^{2}-\gamma\left\|I^{\prime}\left(u_{k}\right)\right\|_{M}\left\|u_{k}\right\|_{M}
$$

we can get that $\left\|u_{k}\right\|_{M}$ is bounded with respect to $k$, that is, $\left\|u_{k}(x)\right\|_{H_{0}^{M}(\Omega)}$ is bounded with respect to $k$.

Denote $S=\frac{2 n p}{n+2 M}$. By $1<p<\frac{n+2 M}{n-2 M}$ it concludes that $1<s<\frac{2 n}{n+2 M} \frac{n+2 M}{n-2 M}=2_{M}^{*}$. Since $\left\|u_{k}(x)\right\|_{H_{0}^{M}(\Omega)}$ is bounded with respect to $k$, by the Sobolev compact imbedding theorem, we can choose a subsequence $\left\{u_{k_{j}}(x)\right\}_{i=1}^{+\infty}$ of $\left\{u_{k}\right\}_{k=1}^{+\infty}$ such that $\left\{u_{k_{j}}(x)\right\}_{i=1}^{+\infty}$ is strongly convergent in $L^{S}(\Omega)$. That is, there exists a function $u^{*}(x) \in L^{s}(\Omega)$ such that when $j \rightarrow+\infty$, $\left\|u_{k_{j}}(x)-u^{*}(x)\right\|_{L^{s}(\Omega)} \rightarrow 0$.

Since $f \in C^{1}, f$ is continuous, by

$$
\left|f\left(u_{k_{j}}(x)\right)\right| \leq a\left|u_{k_{j}}(x)\right|^{p} \quad\left(1<p<\frac{n+2 M}{n-2 M}\right)
$$

we conclude that

$$
\left|f\left(u_{k_{j}}(x)\right)\right|^{\frac{2 n}{n+2 M}} \leq a^{\frac{2 n}{n+2 M}}\left|u_{k_{j}}(x)\right|^{s}
$$


Since $u_{k_{j}}(x) \in L^{s}(\Omega)$, from the above inequality we can get that $f\left(u_{k_{j}}(x)\right) \in L^{\frac{2 n}{n+2 M}}(\Omega)$. Therefore, $f$ is a mapping from $L^{s}(\Omega)$ to $L^{\frac{2 n}{n+2 M}}(\Omega)$. The function $f(u)$ is continuous with respect to $u$, by Theorem 1.1 in [6] the mapping $f: L^{s}(\Omega) \rightarrow L^{\frac{2 n}{n+2 M}}(\Omega)$ is continuous. By $\left\|u_{k_{j}}(x)-u^{*}(x)\right\|_{L^{s}(\Omega)} \rightarrow 0$ as $j \rightarrow+\infty$, it concludes that $\left\|f\left(u_{k_{j}}(x)\right)-f\left(u^{*}(x)\right)\right\|_{L^{\frac{2 n}{n+2 M}(\Omega)}} \rightarrow 0$ as $j \rightarrow+\infty$.

Therefore, the sequence $\left\{f\left(u_{k_{j}}(x)\right)\right\}_{i=1}^{+\infty}$ is a Cauchy sequence in $L^{\frac{2 n}{n+2 M}}(\Omega)$, we have $\left\|f\left(u_{k_{i}}(x)\right)-f\left(u_{k_{j}}(x)\right)\right\|_{\frac{2 n}{n+2 M}(\Omega)} \rightarrow 0$ as $i, j \rightarrow+\infty$.

By $\left(I^{\prime}(u), v\right)_{M}=(u, v)_{M}-\int_{\Omega} f(u) v d x\left(u, v \in H_{0}^{M}(\Omega)\right)$, we can get that

$$
\left(I^{\prime}\left(u_{k_{i}}\right), v\right)_{M}=\left(u_{k_{i}}, v\right)_{M}-\int_{\Omega} f\left(u_{k_{i}}\right) v d x
$$

and

$$
\left(I^{\prime}\left(u_{k_{j}}\right), v\right)_{M}=\left(u_{k_{j}}, v\right)_{M}-\int_{\Omega} f\left(u_{k j}\right) v d x
$$

Hence, we have

$$
\left(u_{k_{i}}-u_{k_{j}}, v\right)_{M}=\left(I^{\prime}\left(u_{k_{i}}\right)-I^{\prime}\left(u_{k_{j}}\right), v\right)+\int_{\Omega}\left[f\left(u_{k_{i}}\right)-f\left(u_{k_{j}}\right)\right] v d x
$$

Then we can get the following estimates:

$$
\begin{aligned}
& \left|\left(u_{k_{i}}-u_{k_{j}}, v\right)_{M}\right| \\
& \quad \leq\left\|I^{\prime}\left(u_{k_{i}}\right)-I^{\prime}\left(u_{k_{j}}\right)\right\|_{M}\|v\|_{M}+\left\|f\left(u_{k_{i}}\right)-f\left(u_{k_{j}}\right)\right\|_{L^{\frac{2 n}{n+2 M}(\Omega)}}\|v\|_{L^{\frac{2 n}{n-2 M}(\Omega)}} \\
& \quad \leq\left\|I^{\prime}\left(u_{k_{i}}\right)-I^{\prime}\left(u_{k_{j}}\right)\right\|_{M}\|v\|_{M}+\left\|f\left(u_{k_{i}}\right)-f\left(u_{k_{j}}\right)\right\|_{L^{\frac{2 n}{n+2 M(}(\Omega)}} C\|v\|_{H_{0}^{M}(\Omega)} \\
& \quad \leq\left\|I^{\prime}\left(u_{k_{i}}\right)-I^{\prime}\left(u_{k_{j}}\right)\right\|_{M}\|v\|_{M}+\left\|f\left(u_{k_{i}}\right)-f\left(u_{k_{j}}\right)\right\|_{L^{\frac{2 n}{n+2 M}(\Omega)}} C\|v\|_{M} .
\end{aligned}
$$

Therefore,

$$
\begin{aligned}
& \left\|u_{k_{i}}-u_{k_{j}}\right\|_{M} \\
& \quad=\sup _{\|v\|_{M \leq 1}}\left|\left(u_{k_{i}}-u_{k_{j}}, v\right)_{M}\right| \\
& \quad \leq\left\|I^{\prime}\left(u_{k_{i}}\right)-I^{\prime}\left(u_{k_{j}}\right)\right\|_{M}+C\left\|f\left(u_{k_{i}}\right)-f\left(u_{k_{j}}\right)\right\|_{L^{\frac{2 n}{n+2 M}(\Omega)}} .
\end{aligned}
$$

When $k \rightarrow+\infty, I^{\prime}\left(u_{k}\right) \rightarrow 0$ in $H_{0}^{M}(\Omega)$, it concludes that when $i, j \rightarrow+\infty$,

$$
\left\|I^{\prime}\left(u_{k_{i}}\right)-I^{\prime}\left(u_{k_{j}}\right)\right\|_{M} \rightarrow 0
$$

By the above discussion we know that $\left\|f\left(u_{k_{i}}\right)-f\left(u_{k_{j}}\right)\right\|_{L^{\frac{2 n}{n+2 M}(\Omega)}} \rightarrow 0$ as $i, j \rightarrow+\infty$. Therefore, we get $\left\|u_{k_{i}}-u_{k_{j}}\right\|_{M} \rightarrow 0$ as $i, j \rightarrow+\infty$. That is, the subsequence $\left\{u_{k_{j}}(x)\right\}_{j=1}^{+\infty}$ is a Cauchy sequence in $H_{0}^{M}(\Omega)$. Since $H_{0}^{M}(\Omega)$ is complete, there exists a function $u(x) \in H_{0}^{M}(\Omega)$ such that

$$
\lim _{j \rightarrow+\infty}\left\|u_{k_{j}}(x)-u(x)\right\|_{H_{0}^{M}(\Omega)}=0
$$


The proof of Step 2 is completed.

Step 3: In what follows we will verify other conditions of mountain pass theorem.

The functional

$$
I(u)=\frac{1}{2} \int_{\Omega}\left|\nabla^{M} u\right|^{2} d x-\int_{\Omega} F(u) d x,
$$

by $F(0)=0$ it concludes that $I(0)=0$.

Assume that $u(x) \in H_{0}^{M}(\Omega)$ and $\|u(x)\|_{M}=r$, where $r$ is a positive constant which will be determined later.

The functional $I(u)=I_{1}(u)-I_{2}(u)=\frac{1}{2} r^{2}-I_{2}(u)$. By $p+1<\frac{n+2 M}{n-2 M}+1=\frac{2 n}{n-2 M}=2_{M}^{*}$, we can get the following estimates:

$$
\begin{aligned}
\left|I_{2}(u)\right| & =\left|\int_{\Omega} F(u) d x\right| \\
& \leq \int_{\Omega}|F(u)| d x \\
& \leq \int_{\Omega} a^{\prime}|u|^{p+1} d x \\
& =a^{\prime}\|u\|_{L^{p+1}(\Omega)}^{p+1} \\
& \leq C\|u\|_{H_{0}^{M}(\Omega)}^{p+1} \\
& \leq C\|u\|_{M}^{p+1} .
\end{aligned}
$$

Hence,

$$
\begin{aligned}
I(u) & =\frac{1}{2} r^{2}-I_{2}(u) \\
& \geq \frac{1}{2} r^{2}-C\|u\|_{M}^{p+1} \\
& =\frac{1}{4} r^{2}+\frac{1}{4} r^{2}-C r^{p+1} .
\end{aligned}
$$

By $p>1$, it concludes that $p+1>2$. Take $r$ sufficiently small such that

$$
\frac{r^{2}}{4}-c r^{p+1} \geq 0
$$

therefore, $I(u) \geq \frac{r^{2}}{4}=a^{\prime \prime}>0$.

Choose function $u(x) \in H_{0}^{M}(\Omega)$ and $u(x) \neq 0$. Denote $v=t u$, where $t$ is a positive constant which will be determined later. We obtain

$$
\begin{aligned}
I(v) & =I_{1}(t u)-I_{2}(t u) \\
& =t^{2} I_{1}(u)-\int_{\Omega} F(t u) d x
\end{aligned}
$$




$$
\begin{aligned}
& \leq t^{2} I_{1}(u)-\int_{\Omega} b^{\prime}|t u|^{p+1} d x \\
& =t^{2} I_{1}(u)-b^{\prime} t^{p+1} \int_{\Omega}|u|^{p+1} d x .
\end{aligned}
$$

Since $p+1>2$, take $t$ sufficiently large such that $I(v)<0$.

Summarize the above discussion, all the conditions of mountain pass theorem are satisfied. By the conclusion of mountain pass theorem, there exist a function $u(x) \in H_{0}^{M}(\Omega)$ and $u(x) \neq 0$ such that $I^{\prime}(u)=0$. That is, for each $v(x) \in H_{0}^{M}(\Omega)$, we have

$$
\left(I^{\prime}(u), v\right)_{M}=0
$$

Therefore, for each $v(x) \in H_{0}^{M}(\Omega)$, the equality

$$
\int_{\Omega}\left(\nabla^{M} u(x)\right)\left(\nabla^{M} v(x)\right) d x-\int_{\Omega} f(u) v d x=0
$$

holds.

The above equality means that $u(x) \in H_{0}^{M}(\Omega)$ is the nontrivial weak solution (nonzero weak solution) to problem (2.1)

$$
\left\{\begin{array}{l}
(-1)^{M} \Delta^{M} u=f(u), \quad x \in \Omega \subset \mathbb{R}^{n}, \\
\left.D^{\gamma} u(x)\right|_{\partial \Omega}=0, \quad 0 \leq|\gamma| \leq M-1 .
\end{array}\right.
$$

When $2 M \geq n$, by a similar method, we can prove that there exists a nontrivial weak solution (nonzero weak solution) to problem (2.1).

The proof of Theorem 2.1 is completed.

\section{Some useful lemmas}

In this section, we give some properties about functionals $J(u), I(u)$ and the potential well $W$ with its depth $d$, which will be used in the following discussion.

Lemma 3.1 Assume that $u(x) \in H_{0}^{M}(\Omega),\left\|\nabla^{M} u\right\| \neq 0$, then we have

(1) $\lim _{\lambda \rightarrow+\infty} J(\lambda u)=-\infty, \lim _{\lambda \rightarrow 0} J(\lambda u)=0$.

(2) There exists unique $\bar{\lambda}=\bar{\lambda}(u)>0$ such that $\left.\frac{d}{d \lambda} J(\lambda u)\right|_{\lambda=\bar{\lambda}}=0$, where $\bar{\lambda}=\left(\frac{\left\|\nabla^{M} u\right\|^{2}}{a\|u\|_{p+1}^{p+1}}\right)^{\frac{1}{p-1}}$.

(3) If $\lambda=\bar{\lambda}, \frac{d^{2}}{d \lambda^{2}} J(\lambda u)<0$.

(4) If $0 \leq \lambda \leq \bar{\lambda}$, then $J(\lambda u)$ increases with respect to $\lambda$, if $\bar{\lambda} \leq \lambda<+\infty$, then $J(\lambda u)$ decreases with respect to $\lambda$.

Proof (1) By

$$
J(\lambda u)=\frac{\lambda^{2}}{2}\left\|\nabla^{M} u\right\|^{2}-\frac{a \lambda^{p+1}}{p+1}\|u\|_{p+1}^{p+1}
$$

and $p>1$, conclusion (1) holds. 
(2) An easy calculation shows that

$$
\frac{d}{d \lambda} J(\lambda u)=\lambda\left\|\nabla^{M} u\right\|^{2}-a \lambda^{p}\|u\|_{p+1}^{p+1}=0,
$$

it can be solved that $\bar{\lambda}=\left(\frac{\left\|\nabla^{M} u\right\|^{2}}{a\|u\|_{p+1}^{p+1}}\right)^{\frac{1}{p-1}}$, conclusion (2) holds.

(3) Substituting $\bar{\lambda}=\left(\frac{\left\|\nabla^{M} u\right\|^{2}}{a\|u\|_{p+1}^{p+1}}\right)^{\frac{1}{p-1}}$ into

$$
\frac{d^{2}}{d \lambda^{2}} J(\lambda u)=\left\|\nabla^{M} u\right\|^{2}-a p \lambda^{p-1}\|u\|_{p+1}^{p+1}
$$

we obtain

$$
\left.\frac{d^{2}}{d \lambda^{2}} J(\lambda u)\right|_{\lambda=\bar{\lambda}}=(1-p)\left\|\nabla^{M} u\right\|^{2}<0 \quad(p>1)
$$

Hence, conclusion (3) holds.

(4) From

$$
\frac{d}{d \lambda} J(\lambda u)=\lambda\left\|\nabla^{M} u\right\|^{2}-a \lambda^{p}\|u\|_{p+1}^{p+1}
$$

and

$$
\bar{\lambda}=\left(\frac{\left\|\nabla^{M} u\right\|^{2}}{a\|u\|_{p+1}^{p+1}}\right)^{\frac{1}{p-1}}
$$

by a direct calculation, we have

$$
\frac{d}{d \lambda} J(\lambda u)=\lambda a\|u\|_{p+1}^{p+1}\left(\bar{\lambda}^{p-1}-\lambda^{p-1}\right) \quad(p>1) .
$$

It shows that if $0<\lambda<\bar{\lambda}$, then $\frac{d}{d \lambda} J(\lambda u)>0$; if $\lambda>\bar{\lambda}$, then $\frac{d}{d \lambda} J(\lambda u)<0$. Conclusion (4) holds.

The proof of Lemma 3.1 is completed.

Lemma 3.2 Let $u(x) \in H_{0}^{M}(\Omega),\left\|\nabla^{M} u\right\| \neq 0$.

(1) Then $I\left(\lambda^{*} u\right)=0$ is equivalent to $J\left(\lambda^{*} u\right)=\sup _{\lambda \geq 0} J(\lambda u)$.

(2) The depth of potential well $d$ in (1.7), there is also a computational formula

$$
d=d_{0}=\frac{a^{\frac{2}{1-p}}}{\gamma C_{*}^{\gamma}}
$$

where $\gamma=\frac{2(p+1)}{p-1}(p>1), C_{*}=\sup _{\substack{u(x) \in H_{0}^{M}(\Omega), u \neq 0}} \frac{\|u\|_{p+1}}{\left\|\nabla^{M} u\right\|}$.

Proof (1) If

$$
I\left(\lambda^{*} u\right)=\left(\lambda^{*}\right)^{2}\left\|\nabla^{M} u\right\|^{2}-a\left(\lambda^{*}\right)^{p+1}\|u\|_{p+1}^{p+1}=0,
$$


from this equality it can be solved that

$$
\lambda^{*}=\left(\frac{\left\|\nabla^{M} u\right\|^{2}}{a\|u\|_{p+1}^{p+1}}\right)^{\frac{1}{p-1}}=\bar{\lambda} .
$$

By (4) in Lemma 3.1 we have $J\left(\lambda^{*} u\right)=\sup _{\lambda \geq 0} J(\lambda u)$.

Conversely, if $J\left(\lambda^{*} u\right)=\sup _{\lambda \geq 0} J(\lambda u)$, by (4) in Lemma 3.1

$$
\lambda^{*}=\bar{\lambda}=\left(\frac{\left\|\nabla^{M} u\right\|^{2}}{a\|u\|_{p+1}^{p+1}}\right)^{\frac{1}{p-1}},
$$

by some computations we have $I\left(\lambda^{*} u\right)=0$.

(2) From the definition of $d$ in (1.7), it follows that

$$
\begin{aligned}
d & =\inf _{\substack{u(x) \in H_{0}^{M}(\Omega) \\
u \neq 0}}\left(\sup _{\lambda \geq 0} J(\lambda u)\right) \\
& =\inf _{\substack{u(x) \in H_{0}^{M}(\Omega) \\
u \neq 0}} J(\bar{\lambda} u),
\end{aligned}
$$

where $\bar{\lambda}=\left(\frac{\left\|\nabla^{M} u\right\|^{2}}{a\|u\|_{p+1}^{p+1}}\right)^{\frac{1}{p-1}}$.

$$
\begin{aligned}
& \text { From } J(\lambda u)=\frac{\lambda^{2}}{2}\left\|\nabla^{M} u\right\|^{2}-\frac{a \lambda^{p+1}}{p+1}\|u\|_{p+1}^{p+1} \text { and } \bar{\lambda}=\left(\frac{\left\|\nabla^{M} u\right\|^{2}}{a\|u\|_{p+1}^{p+1}}\right)^{\frac{1}{p-1}} \text {, we get } \\
& J(\bar{\lambda} u)=\frac{\bar{\lambda}^{2}}{2}\left\|\nabla^{M} u\right\|^{2}-\frac{a \bar{\lambda}^{p+1}}{p+1}\|u\|_{p+1}^{p+1} \\
& =\frac{1}{2}\left(\frac{\left\|\nabla^{M} u\right\|^{2}}{a\|u\|_{p+1}^{p+1}}\right)^{\frac{2}{p-1}}\left\|\nabla^{M} u\right\|^{2}-\frac{a}{p+1}\left(\frac{\left\|\nabla^{M} u\right\|^{2}}{a\|u\|_{p+1}^{p+1}}\right)^{\frac{p+1}{p-1}}\|u\|_{p+1}^{p+1} \\
& =\frac{1}{2} \frac{\left\|\nabla^{M} u\right\|^{\frac{4}{p-1}+2}}{a^{\frac{2}{p-1}}\|u\|_{p+1}^{\frac{2(p+1)}{p-1}}}-\frac{1}{p+1} \frac{\left\|\nabla^{M} u\right\|^{\frac{2(p+1)}{p-1}}}{a^{\frac{p+1}{p-1}-1}\|u\|_{p+1}^{\frac{(p+1)}{p-1}-(p+1)}} \\
& =\frac{1}{2} \frac{\left\|\nabla^{M} u\right\|^{\frac{2(p+1)}{p-1}}}{a^{\frac{2}{p-1}}\|u\|_{p+1}^{\frac{2(p+1)}{p-1}}}-\frac{1}{p+1} \frac{\left\|\nabla^{M} u\right\|^{\frac{2(p+1)}{p-1}}}{a^{\frac{2}{p-1}}\|u\|_{p+1}^{\frac{2(p+1)}{p-1}}} \\
& =\frac{p-1}{2(p+1)} \frac{\left\|\nabla^{M} u\right\|^{\frac{2(p+1)}{p-1}}}{a^{\frac{2}{p-1}}\|u\|_{p+1}^{\frac{2(p+1)}{p-1}}} \\
& =\frac{1}{\frac{2(p+1)}{p-1}} \frac{a^{\frac{2}{1-p}}}{\left(\frac{\|u\|_{p+1}}{\left\|\nabla^{M} u\right\|}\right)^{\frac{2(p+1)}{p-1}}} \text {. }
\end{aligned}
$$

Hence,

$$
\begin{aligned}
d & =\inf _{\substack{u(x) \in H_{0}^{M}(\Omega), u \neq 0}} J(\bar{\lambda} u) \\
& =\inf _{\substack{u(x) \in H_{0}^{M}(\Omega), u \neq 0}} \frac{1}{\frac{2(p+1)}{p-1}} \frac{a^{\frac{2}{1-p}}}{\left(\frac{\|u\|_{p+1}}{\left\|\nabla^{M} u\right\|}\right)^{\frac{2(p+1)}{p-1}}}
\end{aligned}
$$




$$
\begin{aligned}
& =\frac{1}{\frac{2(p+1)}{p-1}} \inf _{\substack{u(x) \in H_{0}^{M}(\Omega), u \neq 0}} \frac{a^{\frac{2}{1-p}}}{\left(\frac{\|u\|_{p+1}}{\left\|\nabla^{M} u\right\|}\right)^{\frac{2(p+1)}{p-1}}} \\
& =\frac{1}{\frac{2(p+1)}{p-1}} \frac{a^{\frac{2}{1-p}}}{\left(\sup _{\substack{u(x) \in H_{0}^{M}(\Omega), u \neq 0}} \frac{\|u\|_{p+1}}{\left\|\nabla^{M} u\right\|}\right)^{\frac{2(p+1)}{p-1}}} \\
& =\frac{1}{\gamma} \frac{a^{\frac{2}{1-p}}}{C_{*}^{\gamma}} .
\end{aligned}
$$

The proof of Lemma 3.2 is completed.

\section{The existence of global weak solutions}

In this section, we prove the existence of global weak solutions to problem (1.1)-(1.3) with subcritical energy $E(0)<d$.

Definition 4.1 Function $u=u(x, t)$ is called a global weak solution to problem (1.1)-(1.3) on $Q_{T}=\Omega \times[0, T)$. If $u(x, t) \in L^{\infty}\left(0, T ; H_{0}^{M}(\Omega)\right)$ with $u_{t}(x, t) \in L^{\infty}\left(0, T ; H_{0}^{M}(\Omega)\right)$ satisfies:

(i) for any $v(x) \in H_{0}^{M}(\Omega), t \in[0, T)$,

$$
\begin{array}{r}
(u(x, t), v(x))+\left(\nabla^{M} u(x, t), \nabla^{M} v(x)\right)+\int_{0}^{t}\left(\nabla^{M} u(x, \tau), \nabla^{M} v(x)\right) d \tau \\
=\left(u_{0}(x), v(x)\right)+\left(\nabla^{M} u_{0}(x), \nabla^{M} v(x)\right)+\int_{0}^{t}(f(u(x, \tau)), v(x)) d \tau ;
\end{array}
$$

(ii) $u(x, 0)=u_{0}(x)$ in $H_{0}^{M}(\Omega)$.

Theorem 4.1 Let $f(u)=a|u|^{p-1} u$ and condition $(H)$ hold. If $E(0)<d$, then problem (1.1)(1.3) admits a global weak solution as long as $u_{0}(x) \in W$. Moreover, for all $t \in[0, T)$, $u(x, t) \in W$.

Proof Let $\omega_{j}(x)(j=1,2,3, \ldots)$ be the eigenfunctions of the problem

$$
(-1)^{M} \Delta^{M} \omega_{j}=\lambda_{j} \omega_{j},\left.\quad D^{\gamma} \omega_{j}\right|_{\partial \Omega}=0 \quad(0 \leq|\gamma| \leq M-1) .
$$

Then $\left\{\omega_{j}(x)\right\}_{j=1}^{+\infty}$ is a complete orthonormal system in $L^{2}(\Omega),\left\{\omega_{j}(x)\right\}_{j=1}^{+\infty}$ is also a complete orthogonal system in $H_{0}^{M}(\Omega)$ (see [4] and [30]). We construct an approximate solution to problem (1.1)-(1.3) as follows:

$$
u_{m}(x, t)=\sum_{j=1}^{m} g_{j m}(t) \omega_{j}(x), \quad m=1,2,3, \ldots
$$

By the Galerkin method, $u_{m}(x, t)(m=1,2,3, \ldots)$ satisfies the following initial value problem of nonlinear ordinary differential system:

$$
\begin{aligned}
& \left(u_{m t}, \omega_{j}\right)+\left((-1)^{M} \Delta^{M} u_{m}, \omega_{j}\right)+\left((-1)^{M} \Delta^{M} u_{m t}, \omega_{j}\right)=\left(f\left(u_{m}\right), \omega_{j}\right), \\
& g_{j m}(0)=a_{j m} \quad(j=1,2, \ldots, m) .
\end{aligned}
$$


Since $u_{0}(x) \in H_{0}^{M}(\Omega),\left\{\omega_{j}(x)\right\}_{j=1}^{+\infty}$ construct a complete orthogonal system in $H_{0}^{M}(\Omega)$, we can choose $a_{j m}(j=1,2, \ldots, m)$ such that, when $m \rightarrow+\infty$,

$$
u_{m}(x, 0)=\sum_{j=1}^{m} a_{j m} \omega_{j}(x) \text { is strongly convergent to } u_{0}(x) \text { in } H_{0}^{M}(\Omega)
$$

Multiplying both sides of (4.2) by $g_{j m}^{\prime}(t)$ and summing up from 1 to $m$ with respect to $j$, we obtain

$$
\begin{aligned}
\left(u_{m t},\right. & \left.\sum_{j=1}^{m} g_{j m}^{\prime}(t) \omega_{j}(x)\right)+\left((-1)^{M} \Delta^{M} u_{m t}, \sum_{j=1}^{m} g_{j m}^{\prime}(t) \omega_{j}(x)\right) \\
& +\left((-1)^{M} \Delta^{M} u_{m}, \sum_{j=1}^{m} g_{j m}^{\prime}(t) \omega_{j}(x)\right) \\
= & \left(f\left(u_{m}\right), \sum_{j=1}^{m} g_{j m}^{\prime}(t) \omega_{j}(x)\right) .
\end{aligned}
$$

Since $u_{m t}(x, t)=\sum_{j=1}^{m} g_{j m}^{\prime}(t) \omega_{j}(x)$, that is,

$$
\left(u_{m t}, u_{m t}\right)+\left((-1)^{M} \Delta^{M} u_{m}, u_{m t}\right)+\left((-1)^{M} \Delta^{M} u_{m t}, u_{m t}\right)=\left(f\left(u_{m}\right), u_{m t}\right) .
$$

Integrating by parts with respect to $x$ in (4.5), we get

$$
\left\|u_{m t}(x, t)\right\|^{2}+\left\|\nabla^{M} u_{m t}(x, t)\right\|^{2}+\frac{1}{2} \frac{d}{d t}\left\|\nabla^{M} u_{m}(x, t)\right\|^{2}=\frac{d}{d t} \int_{\Omega} F\left(u_{m}(x, t)\right) d x,
$$

where $F\left(u_{m}\right)=\int_{0}^{u_{m}} f(s) d s$, that is,

$$
\left\|u_{m t}(x, t)\right\|^{2}+\left\|\nabla^{M} u_{m t}(x, t)\right\|^{2}+\frac{d}{d t}\left(\frac{1}{2}\left\|\nabla^{M} u_{m}(x, t)\right\|^{2}-\int_{\Omega} F\left(u_{m}(x, t)\right) d x\right)=0 .
$$

By integrating (4.6) with respect to $t$ over $[0, t)$ and combining

$$
E_{m}(t)=\frac{1}{2}\left\|\nabla^{M} u_{m}(x, t)\right\|^{2}-\int_{\Omega} F\left(u_{m}(x, t)\right) d x
$$

with

$$
E_{m}(0)=\frac{1}{2}\left\|\nabla^{M} u_{m}(x, 0)\right\|^{2}-\int_{\Omega} F\left(u_{m}(x, 0)\right) d x,
$$

we can get that

$$
\int_{0}^{t}\left\|u_{m \tau}(x, \tau)\right\|^{2} d \tau+\int_{0}^{t}\left\|\nabla^{M} u_{m \tau}(x, \tau)\right\|^{2} d \tau+E_{m}(t)=E_{m}(0)
$$

it concludes that

$$
E_{m}(t) \leq E_{m}(0)
$$


By (4.4), condition $(\mathrm{H})$, and the Sobolev imbedding theorem, it shows that when $m \rightarrow$ $+\infty$,

$$
\begin{aligned}
& \left\|\nabla^{M} u_{m}(x, 0)\right\| \rightarrow\left\|\nabla^{M} u_{0}(x)\right\|, \\
& \left\|u_{m}(x, 0)\right\|_{p+1}^{p+1} \rightarrow\left\|u_{0}(x)\right\|_{p+1}^{p+1} .
\end{aligned}
$$

From $u_{0}(x) \in W$, we get that $I\left(u_{0}\right)>0$ and $J\left(u_{0}\right)<d$ or $\left\|\nabla^{M} u_{0}(x)\right\|=0$. If $I\left(u_{0}\right)>0$, $J\left(u_{0}\right)<d$, when $m \rightarrow+\infty$, we have $I\left(u_{m}(x, 0)\right) \rightarrow I\left(u_{0}(x)\right), J\left(u_{m}(x, 0)\right) \rightarrow J\left(u_{0}(x)\right)$. Then, for sufficiently large $m$, the inequalities $I\left(u_{m}(x, 0)\right)>0$ and $J\left(u_{m}(x, 0)\right)<d$ hold, that is, $u_{m}(x, 0) \in W$.

If $\left\|\nabla^{M} u_{0}(x)\right\|=0$, when $m \rightarrow+\infty$, we see that $\left\|\nabla^{M} u_{m}(x, 0)\right\| \rightarrow\left\|\nabla^{M} u_{0}(x)\right\|=0$. Then, for sufficiently large $m, u_{m}(x, 0) \in W$.

In what follows we will prove that, for sufficiently large $m$ and for $t \in[0, T), u_{m}(x, t) \in W$. If the above conclusion is wrong, there is sufficiently large $m$ and $t_{0}=t_{0}(m) \in(0, T)$ such that $u_{m}\left(x, t_{0}\right) \in \partial W$. From $u_{m}\left(x, t_{0}\right) \in \partial W$, it concludes that

$$
I\left(u_{m}\left(x, t_{0}\right)\right)=0, \quad\left\|\nabla^{M} u_{m}\left(x, t_{0}\right)\right\| \neq 0 \quad \text { or } \quad J\left(u_{m}\left(x, t_{0}\right)\right)=d .
$$

By $f(u)=a|u|^{p-1} u$ and $f(u) u \geq 0$, we can get

$$
0 \leq F(u) \leq \frac{a}{p+1}|u|^{p+1} \quad\left(F(u)=\int_{0}^{u} f(s) d s\right),
$$

therefore,

$$
\int_{\Omega} F(u) d x \leq \frac{a}{p+1}\|u\|_{p+1}^{p+1}
$$

that is,

$$
-\int_{\Omega} F(u) d x \geq-\frac{a}{p+1}\|u\|_{p+1}^{p+1}
$$

By a direct calculation, we know that

$$
\begin{aligned}
& \left|\int_{\Omega} F\left(u_{m}(x, 0)\right) d x-\int_{\Omega} F\left(u_{0}(x)\right) d x\right| \\
& \quad=\left|\int_{\Omega} f\left(\varphi_{m}\right)\left(u_{m}(x, 0)-u_{0}(x)\right) d x\right| \\
& \quad \leq\left\|f\left(\varphi_{m}\right)\right\|_{\frac{p+1}{p}}\left\|u_{m}(x, 0)-u_{0}(x)\right\|_{p+1},
\end{aligned}
$$

where $\varphi_{m}=u_{0}(x)+\theta\left(u_{m}(x, 0)-u_{0}(x)\right), 0<\theta<1$.

From $f(u)=a|u|^{p-1} u$, we obtain

$$
\left\|f\left(\varphi_{m}\right)\right\|_{\frac{p+1}{p}}\left\|u_{m}(x, 0)-u_{0}(x)\right\|_{p+1}=\left\|a\left|\varphi_{m}\right|^{p}\right\| \frac{p+1}{p}\left\|u_{m}(x, 0)-u_{0}(x)\right\|_{p+1} .
$$

From (4.4), we can get $\left\|u_{m}(x, 0)-u_{0}(x)\right\|_{H_{0}^{M}(\Omega)} \rightarrow 0$ as $m \rightarrow+\infty$. By the Sobolev imbedding theorem and condition $(\mathrm{H})$, it concludes that $\left\|u_{m}(x, 0)-u_{0}(x)\right\|_{p+1} \rightarrow 0$ as $m \rightarrow+\infty$ and $\left\|u_{m}(x, 0)\right\|_{p+1} \rightarrow\left\|u_{0}(x)\right\|_{p+1}$ as $m \rightarrow+\infty$. 
By the Sobolev imbedding theorem and condition $(\mathrm{H}),\left\|a\left|\varphi_{m}\right|^{p}\right\|_{\frac{p+1}{p}}$ is bounded with respect to $m$. Therefore, when $m \rightarrow+\infty$, we derive

$$
\left|\int_{\Omega} F\left(u_{m}(x, 0)\right) d x-\int_{\Omega} F\left(u_{0}(x)\right) d x\right| \rightarrow 0,
$$

that is,

$$
\int_{\Omega} F\left(u_{m}(x, 0)\right) d x \rightarrow \int_{\Omega} F\left(u_{0}(x)\right) d x \quad \text { as } m \rightarrow+\infty \text {. }
$$

From (4.8), it shows that

$$
\begin{aligned}
E_{m}(0) & =\frac{1}{2}\left\|\nabla^{M} u_{m}(x, 0)\right\|^{2}-\int_{\Omega} F\left(u_{m}(x, 0)\right) d x \\
& \rightarrow \frac{1}{2}\left\|\nabla^{M} u_{0}(x)\right\|^{2}-\int_{\Omega} F\left(u_{0}(x)\right) d x \quad \text { as } m \rightarrow+\infty \\
& =E(0) .
\end{aligned}
$$

By $E(0)<d$, we get that $E_{m}(0)<d$ for sufficiently large $m$. Hence, for $t \in[0, T)$ and for sufficiently large $m$, we can get

$$
\begin{aligned}
\int_{0}^{t} & \left\|u_{m \tau}(x, \tau)\right\|^{2} d \tau+J\left(u_{m}(x, t)\right)+\int_{0}^{t}\left\|\nabla^{M} u_{m \tau}(x, \tau)\right\|^{2} d \tau \\
= & \int_{0}^{t}\left\|u_{m \tau}(x, \tau)\right\|^{2} d \tau+\frac{1}{2}\left\|\nabla^{M} u_{m}(x, t)\right\|^{2} \\
& \quad-\frac{a}{p+1}\left\|u_{m}(x, t)\right\|_{p+1}^{p+1}+\int_{0}^{t}\left\|\nabla^{M} u_{m \tau}(x, \tau)\right\|^{2} d \tau \\
\leq & \int_{0}^{t}\left\|u_{m \tau}(x, \tau)\right\|^{2} d \tau+\frac{1}{2}\left\|\nabla^{M} u_{m}(x, t)\right\|^{2} \\
& -\int_{\Omega} F\left(u_{m}(x, t)\right) d x+\int_{0}^{t}\left\|\nabla^{M} u_{m \tau}(x, \tau)\right\|^{2} d \tau \\
= & \frac{1}{2}\left\|\nabla^{M} u_{m}(x, 0)\right\|^{2}-\int_{\Omega} F\left(u_{m}(x, 0)\right) d x \\
= & E_{m}(0)<d .
\end{aligned}
$$

In the above conclusion, inequality (4.7) has been used.

From inequality (4.9) we can get

$$
J\left(u_{m}\left(x, t_{0}\right)\right)<d
$$

This is a contradiction with the definition of $d$ in (1.7). Since $u_{m}\left(x, t_{0}\right) \in H_{0}^{M}(\Omega)$, $I\left(u_{m}\left(x, t_{0}\right)\right)=0,\left\|\nabla^{M} u_{m}\left(x, t_{0}\right)\right\| \neq 0$, the inequality $J\left(u_{m}\left(x, t_{0}\right)\right) \geq d$ should hold. Hence $I\left(u_{m}\left(x, t_{0}\right)\right)=0$ and $\left\|\nabla^{M} u_{m}\left(x, t_{0}\right)\right\| \neq 0$ do not hold. On the other hand, by inequality (4.9), $J\left(u_{m}\left(x, t_{0}\right)\right)=d$ is impossible. Therefore, for sufficiently large $m$ and for $t \in[0, T)$, $u_{m}(x, t) \in W$, we obtain that $I\left(u_{m}(x, t)\right)>0$ holds. 
Substituting

$$
J\left(u_{m}\right)=\frac{p-1}{2(p+1)}\left\|\nabla^{M} u_{m}\right\|^{2}+\frac{1}{p+1} I\left(u_{m}\right)
$$

into inequality (4.9), we get

$$
\begin{aligned}
& \int_{0}^{t}\left\|u_{m \tau}(x, \tau)\right\|^{2} d \tau+\frac{p-1}{2(p+1)}\left\|\nabla^{M} u_{m}\right\|^{2} \\
& +\frac{1}{p+1} I\left(u_{m}\right)+\int_{0}^{t}\left\|\nabla^{M} u_{m \tau}(x, \tau)\right\|^{2} d \tau<d, \quad t \in[0, T) .
\end{aligned}
$$

By $I\left(u_{m}(x, t)\right)>0$ for $t \in[0, T)$, it shows that

$$
\left\{\begin{array}{l}
\int_{0}^{t}\left\|u_{m \tau}(x, \tau)\right\|^{2} d \tau<d, \quad t \in[0, T), \\
\frac{p-1}{2(p+1)}\left\|\nabla^{M} u_{m}(x, t)\right\|^{2}<d, \quad t \in[0, T), \\
\int_{0}^{t}\left\|\nabla^{M} u_{m \tau}(x, \tau)\right\|^{2} d \tau<d, \quad t \in[0, T) .
\end{array}\right.
$$

Therefore, $\left\{u_{m}(x, t)\right\}_{m=1}^{+\infty}$ is bounded in $L^{\infty}\left(0, T ; H_{0}^{M}(\Omega) \cap L^{p+1}(\Omega)\right.$ ) (p satisfies condition $(\mathrm{H}))$ and $\left\{u_{m t}(x, t)\right\}$ is bounded in $L^{2}\left(0, T ; H_{0}^{M}(\Omega)\right)$. By $f(u)=a|u|^{p-1} u$, it shows that $\left\{f\left(u_{m}\right)\right\}_{m=1}^{+\infty}$ is bounded in $L^{\infty}\left(0, T ; L^{q}(\Omega)\right)\left(q=\frac{p+1}{p}\right)$.

Since $\int_{\Omega}\left|f\left(u_{m}\right)\right|^{2} d x=a^{2} \int_{\Omega}\left|u_{m}\right|^{2 p} d x$, from the second inequality in (4.10), we can get that $\left\|u_{m}\right\|_{H_{0}^{M}(\Omega)}$ is uniformly bounded with respect to $m$. By condition $(\mathrm{H})$ and the Sobolev imbedding theorem, $\int_{\Omega}\left|u_{m}\right|^{2 p} d x$ is uniformly bounded with respect to $m$, it concludes that $\left\|f\left(u_{m}\right)\right\|$ is uniformly bounded with respect to $m$.

Since $\left\{\omega_{j}(x)\right\}_{j=1}^{+\infty}$ is a complete orthonormal system in $L^{2}(\Omega),\left\|\omega_{j}(x)\right\|_{L^{2}(\Omega)}=\left\|\omega_{j}(x)\right\|=1$ $(j=1,2,3, \ldots)$, then $\left|\left(f\left(u_{m}\right), \omega_{j}(x)\right)\right| \leq\left\|f\left(u_{m}\right)\right\| \cdot\left\|\omega_{j}(x)\right\|=\left\|f\left(u_{m}\right)\right\|$ is uniformly bounded with respect to $m$. Hence, for $t \in[0, T),\left|\left(f\left(u_{m}\right), \omega_{j}(x)\right)\right|(j=1,2, \ldots, m)$ are uniformly bounded with respect to $m$.

By the theory of ordinary differential system, there exists a global solution

$$
u_{m}(x, t)=\sum_{j=1}^{m} g_{j m}(t) \omega_{j}(x)
$$

to problem (4.2)-(4.3) on [0,T). Therefore, by the compactness principle, there exist functions $u(x, t), X(x, t)$ and the subsequence of $\left\{u_{m}(x, t)\right\}_{m=1}^{+\infty}$ (still denoted by $\left.\left\{u_{m}(x, t)\right\}_{m=1}^{+\infty}\right)$ such that when $m \rightarrow+\infty, u_{m}(x, t) \rightarrow u(x, t)$ in $L^{\infty}\left(0, T ; H_{0}^{M}(\Omega) \cap L^{p+1}(\Omega)\right)$ weakly-star, $f\left(u_{m}(x, t)\right) \rightarrow X(x, t)$ in $L^{\infty}\left(0, T ; L^{q}(\Omega)\right)$ weakly-star $\left(q=\frac{p+1}{p}\right)$.

By the first inequality of (4.10) it shows that $\left\{u_{m t}(x, t)\right\}_{m=1}^{+\infty}$ is bounded in $L^{2}\left(Q_{T}\right)$. Since $\left\|\nabla^{M} u_{m}\right\|$ is an equivalent norm in $H_{0}^{M}(\Omega)$, by the second inequality of (4.10) it concludes that $\left\{u_{m}(x, t)\right\}_{m=1}^{+\infty}$ and $\left\{\nabla u_{m}(x, t)\right\}_{m=1}^{+\infty}$ are bounded in $L^{2}\left(Q_{T}\right)$. Hence $\left\{u_{m}(x, t)\right\}_{m=1}^{+\infty}$ is bounded in $H^{1}\left(Q_{T}\right)\left(Q_{T}=\Omega \times[0, T)\right)$.

Since $H^{1}\left(Q_{T}\right)$ can be compactly imbedded into $L^{2}\left(Q_{T}\right)$, there exists a subsequence of $\left\{u_{m}(x, t)\right\}_{m=1}^{+\infty}$ (still denoted by $\left\{u_{m}(x, t)\right\}_{m=1}^{+\infty}$ ) such that when $m \rightarrow+\infty, u_{m}(x, t)$ is strongly convergent to $u(x, t)$ in $L^{2}\left(Q_{T}\right)$ and $u_{m}(x, t)$ is almost everywhere convergent to $u(x, t)$ in $Q_{T}$. 
Since when $m \rightarrow+\infty, u_{m}(x, t)$ is almost everywhere convergent to $u(x, t)$ in $Q_{T}$ and $\left\{f\left(u_{m}(x, t)\right)\right\}_{m=1}^{+\infty}$ is bounded in $L^{\infty}\left(0, T ; L^{q}(\Omega)\right)\left(q=\frac{p+1}{p}\right) .\left\{f\left(u_{m}(x, t)\right)\right\}_{m=1}^{+\infty}$ is also bounded in $L^{q}\left(Q_{T}\right)$. By Lemma 1.3 in [11], we can get that $f\left(u_{m}(x, t)\right)$ is weakly convergent to $f(u(x, t))$ in $L^{q}\left(Q_{T}\right)$ as $m \rightarrow+\infty$.

For $t \in[0, T)$, integrating (4.2) with respect to $t$ and integrating by parts with respect to $x$, we get the following equality:

$$
\begin{aligned}
& \left(u_{m}(x, t), \omega_{j}(x)\right)+\left(\nabla^{M} u_{m}(x, t), \nabla^{M} \omega_{j}(x)\right)+\int_{0}^{t}\left(\nabla^{M} u_{m}(x, \tau), \nabla^{M} \omega_{j}(x)\right) d \tau \\
& =\left(u_{m}(x, 0), \omega_{j}(x)\right)+\left(\nabla^{M} u_{m}(x, 0), \nabla^{M} \omega_{j}(x)\right) \\
& \quad+\int_{0}^{t}\left(f\left(u_{m}(x, \tau)\right), \omega_{j}(x)\right) d \tau \quad(j=1,2,3, \ldots) .
\end{aligned}
$$

Let $m \rightarrow+\infty$, notice that $u_{m}(x, 0)$ is strongly convergent to $u_{0}(x)$ in $H_{0}^{M}(\Omega), u_{m}(x, t) \rightarrow$ $u(x, t)$ in $L^{\infty}\left(0, T ; H_{0}^{M}(\Omega)\right)$ weakly-star, and $f\left(u_{m}(x, t)\right)$ is weakly convergent to $f(u(x, t))$ in $L^{q}\left(Q_{T}\right)$. Hence, the following equality

$$
\begin{aligned}
& \left(u(x, t), \omega_{j}(x)\right)+\left(\nabla^{M} u(x, t), \nabla^{M} \omega_{j}(x)\right)+\int_{0}^{t}\left(\nabla^{M} u(x, \tau), \nabla^{M} \omega_{j}(x)\right) d \tau \\
& \quad=\left(u_{0}(x), \omega_{j}(x)\right)+\left(\nabla^{M} u_{0}(x), \nabla^{M} \omega_{j}(x)\right)+\int_{0}^{t}\left(f(u(x, \tau)), \omega_{j}(x)\right) d \tau \quad(j=1,2,3, \ldots)
\end{aligned}
$$

holds.

Because $\left\{\omega_{j}(x)\right\}_{j=1}^{+\infty}$ is a complete orthogonal system in $H_{0}^{M}(\Omega)$, for any $v(x) \in H_{0}^{M}(\Omega)$, we get

$$
\begin{aligned}
& (u(x, t), v(x))+\left(\nabla^{M} u(x, t), \nabla^{M} v(x)\right)+\int_{0}^{t}\left(\nabla^{M} u(x, \tau), \nabla^{M} v(x)\right) d \tau \\
& \quad=\left(u_{0}(x), v(x)\right)+\left(\nabla^{M} u_{0}(x), \nabla^{M} v(x)\right)+\int_{0}^{t}(f(u(x, \tau)), v(x)) d \tau, \quad t \in[0, T) .
\end{aligned}
$$

In what follows we prove that the initial condition $u(x, 0)=u_{0}(x)$ holds.

By the second inequality in (4.10), when $m \rightarrow+\infty, u_{m}(x, t) \rightarrow u(x, t)$ in $L^{\infty}(0, T$; $\left.H_{0}^{M}(\Omega)\right) \subset L^{2}\left(0, T ; H_{0}^{M}(\Omega)\right)$ weakly-star. By the third inequality in (4.10), when $m \rightarrow+\infty$, $u_{m t}(x, t) \rightarrow u_{t}(x, t)$ in $L^{2}\left(0, T ; H_{0}^{M}(\Omega)\right)$ weakly-star. According to Lemma 1.2 in [11] it shows that

$$
u_{m}(x, t), \quad u(x, t) \in C\left(0, T ; H_{0}^{M}(\Omega)\right) .
$$

Therefore, when $m \rightarrow+\infty$,

$$
u_{m}(x, 0) \rightarrow u(x, 0) \quad \text { in } H_{0}^{M}(\Omega) \text { weakly-star. }
$$

From (4.4) we know that when $m \rightarrow+\infty, u_{m}(x, 0)$ is strongly convergent to $u_{0}(x)$ in $H_{0}^{M}(\Omega)$, we can get that $u(x, 0)=u_{0}(x)$. According to the definition of global weak solution, $u(x, t)$ is the global weak solution to problem (1.1)-(1.3) in $Q_{T}$.

Lastly, we will prove that, for $t \in[0, T), u(x, t) \in W$. 
In what follows we use the method of contradiction to prove that, for $t \in[0, T)$, $u(x, t) \in W$. If there exists $t_{0} \in(0, T)$ such that if $t \in\left[0, t_{0}\right), u(x, t) \in W=\{u(x) \mid u(x) \in$ $\left.H_{0}^{M}(\Omega), I(u)>0, J(u)<d\right\} \cup\{0\}$, but $u\left(x, t_{0}\right) \in \partial W$. From $u\left(x, t_{0}\right) \in \partial W$, we see that $u\left(x, t_{0}\right) \in$ $H_{0}^{M}(\Omega), I\left(u\left(x, t_{0}\right)\right)=0$, and $\left\|\nabla^{M} u\left(x, t_{0}\right)\right\| \neq 0$ or $J\left(u\left(x, t_{0}\right)\right)=d$.

Making $L^{2}(\Omega)$ inner product by $u_{t}(x, t)$ in both sides of equation

$$
u_{t}+(-1)^{M} \Delta^{M} u_{t}+(-1)^{M} \Delta^{M} u=f(u)
$$

we get

$$
\left(u_{t}, u_{t}\right)+\left(u_{t},(-1)^{M} \Delta^{M} u_{t}\right)+\left(u_{t},(-1)^{M} \Delta^{M} u\right)=\left(u_{t}, f(u)\right)
$$

Integrating by parts with respect to $x$, it concludes that

$$
\begin{gathered}
\left(u_{t}, u_{t}\right)+\left(\nabla^{M} u_{t}, \nabla^{M} u_{t}\right)+\frac{1}{2} \frac{d}{d t}\left(\nabla^{M} u, \nabla^{M} u\right) \\
=\frac{d}{d t} \int_{\Omega} F(u) d x \quad\left(F(u)=\int_{0}^{u} f(s) d s\right) .
\end{gathered}
$$

For all $t \in[0, T)$, integrating from 0 to $t$ with respect to $t$, we obtain

$$
\begin{aligned}
& {\left[u_{t}, u_{t}\right]+\left[\nabla^{M} u_{t}, \nabla^{M} u_{t}\right]+\frac{1}{2}\left(\nabla^{M} u(x, t), \nabla^{M} u(x, t)\right)-\frac{1}{2}\left(\nabla^{M} u(x, 0), \nabla^{M} u(x, 0)\right)} \\
& \quad=\int_{\Omega} F(u(x, t)) d x-\int_{\Omega} F(u(x, 0)) d x
\end{aligned}
$$

That is,

$$
\begin{aligned}
& {\left[u_{t}, u_{t}\right]+\left[\nabla^{M} u_{t}, \nabla^{M} u_{t}\right]+\frac{1}{2}\left\|\nabla^{M} u(x, t)\right\|^{2}-\frac{1}{2}\left\|\nabla^{M} u_{0}(x)\right\|^{2}} \\
& =\int_{\Omega} F(u(x, t)) d x-\int_{\Omega} F\left(u_{0}(x)\right) d x,
\end{aligned}
$$

where $\left[u_{t}, u_{t}\right]=\int_{0}^{t}\left(u_{\tau}(x, \tau), u_{\tau}(x, \tau)\right) d \tau,\left[\nabla^{M} u_{t}, \nabla^{M} u_{t}\right]=\int_{0}^{t}\left(\nabla^{M} u_{\tau}(x, \tau), \nabla^{M} u_{\tau}(x, \tau)\right) d \tau$.

By some computations, we get

$$
\begin{aligned}
& {\left[u_{t}, u_{t}\right]+\left[\nabla^{M} u_{t}, \nabla^{M} u_{t}\right]+\frac{1}{2}\left\|\nabla^{M} u(x, t)\right\|^{2}-\int_{\Omega} F(u(x, t)) d x} \\
& =\frac{1}{2}\left\|\nabla^{M} u_{0}(x)\right\|^{2}-\int_{\Omega} F\left(u_{0}(x)\right) d x .
\end{aligned}
$$

Combining

$$
E(t)=\frac{1}{2}\left\|\nabla^{M} u(x, t)\right\|^{2}-\int_{\Omega} F(u(x, t)) d x
$$

with

$$
E(0)=\frac{1}{2}\left\|\nabla^{M} u_{0}(x)\right\|^{2}-\int_{\Omega} F\left(u_{0}(x)\right) d x,
$$


we obtain

$$
\left[u_{t}, u_{t}\right]+\left[\nabla^{M} u_{t}, \nabla^{M} u_{t}\right]+E(t)=E(0), \quad t \in[0, T),
$$

it concludes that

$$
E(t) \leq E(0), \quad t \in[0, T)
$$

By

$$
\begin{aligned}
& E(t)=\frac{1}{2}\left\|\nabla^{M} u(x, t)\right\|^{2}-\int_{\Omega} F(u(x, t)) d x, \\
& J(u(x, t))=\frac{1}{2}\left\|\nabla^{M} u(x, t)\right\|^{2}-\frac{a}{p+1}\|u(x, t)\|_{p+1}^{p+1},
\end{aligned}
$$

and inequality (4.7), we arrive at

$$
\frac{1}{2}\left\|\nabla^{M} u\right\|^{2}-\frac{a}{p+1}\|u\|_{p+1}^{p+1} \leq \frac{1}{2}\left\|\nabla^{M} u\right\|^{2}-\int_{\Omega} F(u) d x .
$$

Therefore, for $t \in[0, T)$, we have

$$
\begin{aligned}
J(u(x, t)) & =\frac{1}{2}\left\|\nabla^{M} u(x, t)\right\|^{2}-\frac{a}{p+1}\|u(x, t)\|_{p+1}^{p+1} \\
& \leq \frac{1}{2}\left\|\nabla^{M} u(x, t)\right\|^{2}-\int_{\Omega} F(u(x, t)) d x \\
& =E(t) \leq E(0)<d .
\end{aligned}
$$

By inequality (4.11) it shows that $J\left(u\left(x, t_{0}\right)\right)=d$ is impossible. From the definition of $d$ in (1.7), by $u\left(x, t_{0}\right) \in H_{0}^{M}(\Omega), I\left(u\left(x, t_{0}\right)\right)=0$, and $\left\|\nabla^{M} u\left(x, t_{0}\right)\right\| \neq 0$, we know that $J\left(u\left(x, t_{0}\right)\right) \geq d$. On the other hand, from inequality (4.11), we see that $J\left(u\left(x, t_{0}\right)\right)<d$. This is a contradiction! Therefore, $I\left(u\left(x, t_{0}\right)\right)=0$ and $\left\|\nabla^{M} u\left(x, t_{0}\right)\right\| \neq 0$ do not hold. Summarizing the above discussion, we have proved that, for $t \in[0, T), u(x, t) \in W$.

The proof of Theorem 4.1 is completed.

\section{Acknowledgements}

Authors are greatly indebted to the editor and the referees who contributed by giving insightful comments and wise advices.

\section{Funding}

This paper is supported by Guangdong Provincial Department of Education 2018 Teaching Team Project (No. 99161010120)

\section{Abbreviations}

Not applicable.

\section{Availability of data and materials}

Date sharing not applicable to this article as no datasets were generated or analysed during the current study.

\section{Competing interests}

The authors declare that they have no competing interests. 


\section{Publisher's Note}

Springer Nature remains neutral with regard to jurisdictional claims in published maps and institutional affiliations.

\section{Received: 15 September 2020 Accepted: 17 December 2020 Published online: 09 January 2021}

\section{References}

1. Benedetto, E.D., Pierre, M.: On the maximum principle for pseudoparabolic equations. Indiana Univ. Math. J. 30, 821-854 (1981)

2. Benjamin, T.B., Bona, J.L., Mahony, J.J.: Model equations for long waves in nonlinear dispersive systems. Philos. Trans. R. Soc. Lond. Ser. A 272, 47-78 (1972)

3. Cao, Y., Yin, J.X., Wang, C.P.: Cauchy problems of semilinear pseudo-parabolic equations. J. Differ. Equ. 246, 4568-4590 (2009)

4. Folland, G.B.: Introduction to Partial Differential Equation, 2nd edn. Princeton University Press, Princeton (1995)

5. Gopala Rao, V.R., Ting, T.W.: Solutions of pseudo-heat equations in the whole space. Arch. Ration. Mech. Anal. 49, 57-78 (1972)

6. Guo, D.J.: Nonlinear Functional Analysis, 3rd edn. Higher Eduction Press, Beijing (2015) (in Chinese)

7. Korpusov, M.O., Sveshnikov, A.G.: Three-dimensional nonlinear evolution equations of pseudoparabolic type in problems of mathematical physics. Comput. Math. Math. Phys. 43, 1765-1797 (2003)

8. Korpusov, M.O., Sveshnikov, A.G.: Blow-up of solutions of Sobolev-type nonlinear equations with cubic sources. J. Differ. Equ. 42, 431-443 (2006)

9. Lian, W., Xu, R.Z.: Global well-posedness of nonlinear wave equation with weak and strong damping terms and logarithmic source term. Adv. Nonlinear Anal. 9(1), 613-632 (2020)

10. Lian, W., Xu, R.Z., Radulescu, V.D., Yang, Y.B., Zhao, N.: Global well-posedness for a class of fourth order nonlinear strongly damped wave equations. Adv. Calc. Var. (2019). https://doi.org/10.1515/acv-2019-0039

11. Lions, J.L.: Some Methods for Solving Nonlinear Boundary Value Problems. Translated by Boling Guo. Zhongshan University Press (1992)

12. Liu, Y.C., Xu, R.Z., Yu, T.: Global existence, nonexistence and asymptotic behavior of solutions for the Cauchy problem of semilinear heat equations. Nonlinear Anal. 68, 3332-3348 (2008)

13. Liu, Y.C., Zhao, J.S.: On potential wells and applications to semilinear hyperbolic equations and parabolic equations. Nonlinear Anal. 64, 2665-2687 (2006)

14. Padron, V:: Effect of aggregation on population recovery modeled by a forward-backward pseudoparabolic equation. Trans. Am. Math. Soc. 356, 2739-2756 (2004)

15. Papageorgiou, N.S., Radulescu, V.D.: Multiple solutions with precise sign for nonlinear parametric Robin problems. J. Differ. Equ. 256(7), 2449-2479 (2014)

16. Papageorgiou, N.S., Radulescu, V.D.: Multiplicity of solutions for resonant Neumann problems with an indefinite and unbounded potential. Trans. Am. Math. Soc. 367(12), 8723-8756 (2015)

17. Papageorgiou, N.S., Radulescu, V.D., Repovs, D.D.: Nonlinear Analysis-Theory and Methods. Springer Monographs Math. Springer, Cham (2019)

18. Payne, L.E., Sattinger, D.H.: Saddle points and instability on nonlinear hyperbolic equations. Isr. J. Math. 22, 273-303 (1975)

19. Sattinger, D.H.: On global solutions of nonlinear hyperbolic equations. Arch. Ration. Mech. Anal. 30, 148-172 (1968)

20. Showalter, R.E., Ting, T.W.: Pseudoparabolic partial differential equations. SIAM J. Math. Anal. 1, 1-26 (1970)

21. Ting, T.W.: Certain non-steady flows of second-order fluids. Arch. Ration. Mech. Anal. 14, 1-26 (1963)

22. Ting, T.W.: Parabolic and pseudo-parabolic partial differential equations. J. Math. Soc. Jpn. 21, 440-453 (1969)

23. Xu, R.Z.: Asymptotic behavior and blow up of solutions for semilinear parabolic equations at critical energy level. Math. Comput. Simul. 80, 808-813 (2009)

24. $\mathrm{Xu}, \mathrm{R} . Z$.: Initial boundary value problem for semilinear hyperbolic equations and parabolic equations with critical initial data. Q. Appl. Math. 3, 459-468 (2010)

25. Xu, R.Z., Lian, W., Niu, Y.: Global well-posedness of coupled parabolic systems. Sci. China Math. 63, 321-356 (2020)

26. Xu, R.Z., Su, J.: Global existence and finite time blow-up for a class of semilinear pseudo-parabolic equations. J. Funct. Anal. 264, 2732-2763 (2013)

27. Xu, R.Z., Wang, X.C., Yang, Y.B.: Blowup and blowup time for a class of semilinear pseudo-parabolic equations with high initial energy. Appl. Math. Lett. 83, 176-181 (2018)

28. Xu, R.Z., Wang, X.C., Yang, Y.B., Chen, S.H.: Global solutions and finite time blow-up for fourth order nonlinear damped wave equation. J. Math. Phys. 56(6), 1-27 (2018)

29. Xu, R.Z., Zhang, M.Y., Chen, S.H., Yang, Y.B., Shen, J.H.: The initial-boundary value problems for a class of six order nonlinear wave equation. Discrete Contin. Dyn. Syst. 37(11), 5631-5649 (2017)

30. Yan, M., Xiao, L.M.: The initial boundary value problems for a class of multidimensional nonlinear pseudo-hyperbolic system of higher order. Adv. Appl. Math. 2(1), 20-33 (2013) (in Chinese) 